\title{
INNOVATIVE CONCEPT OF SYNERGETIC AIR TRAFFIC MANAGEMENT UNDER POLYCONFLICTS
}

\author{
Volodymyr KHARCHENKO ${ }^{1}$, Valeriy CHEPIZHENKO ${ }^{2}$, Svetlana PAVLOVA ${ }^{3}$, Wang BO ${ }^{4}$ \\ 1, 2, 3 Air Navigation Systems Department, Air Navigation Institute, National Aviation University, \\ Kosmonavta Komarova ave. 1, Kiev, 03680, Ukraine \\ ${ }^{4}$ Economics and Management College of Ningbo University of Technology, China \\ E-mails: ${ }^{1}$ kharh@nau.edu.ua; ${ }^{2}$ chiv@nau.edu.ua; \\ 3psv@nau.edu.ua (correspondingauthor); ${ }^{4}$ bo305@hotmail.com
}

Received 20 May 2014; accepted 10 October 2014
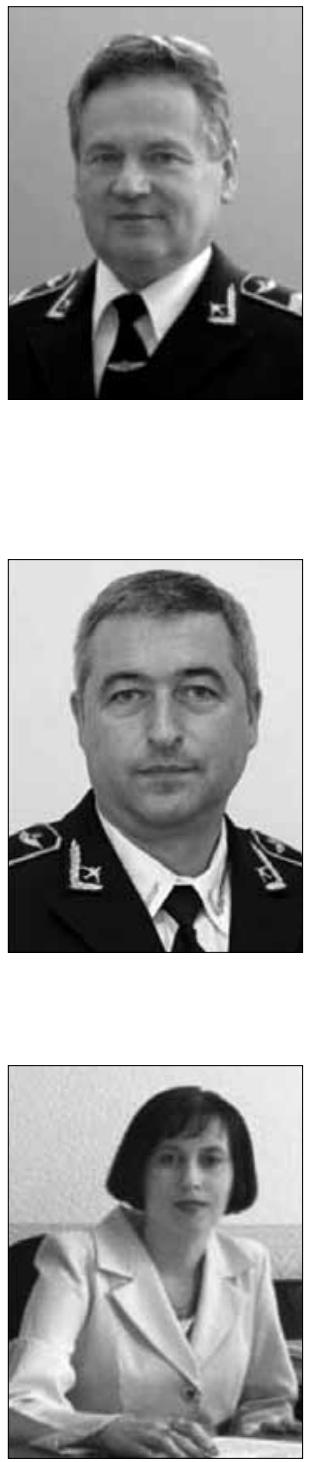

Volodymyr KHARCHENKO, Prof. Dr Sc (Eng)

Date of birth: 1943.

Education: Kiev Civil Aviation Engineers Institute, a degree in Radio Engineering (1962-1967). Affiliations and functions: engineer at United Aircraft Troops (1967-1969), junior and senior researcher (1969-1984), assistant professor (1984-1987), Dr Sc (Eng) (1994), professor (1987-1994) at Kiev International University of Civil Aviation. From 2001 he is the vice-rector for scientific-research work at the National Aviation University.

Research interests: communication; navigation; surveillance.

Publications: author of about 400 scientific papers, monographs, textbooks, training aids, articles and proceedings.

Other: member of Ukrainian Transport Academy (1996), Russian Academy of Navigation and Control (1999), Institute of Engineers of Electrics and Electronics in USA (2006).

Valeriy CHEPIZHENKO, Prof. Dr Sc (Eng)

Date of birth: 1968.

Education: Kiev Higher Military Aviation Engineering School with a degree in Electrical Engineering (1985-1990).

Affiliations and functions: served in the army (1990-2010), postgraduate student (19961999), associate professor (2010-2013), Dr Sc (Eng) (2013), Director of Air Navigation Institute (2013 - to present) in the National Aviation University (Ukraine).

Research interests: synergetic aircraft polyconflict resolution methods; methods of integrated dynamics and technical maintenance of an aircraft in the CNS/ATM environment.

Publications: author of about 60 scientific papers, monographs, textbooks, training aids, articles and proceedings.

Svetlana PAVLOVA, Prof. Dr Sc (Eng)

Date of birth: 1966.

Education: Kiev Construction Institute with a degree in System Engineering (1983-1988). Affiliations and functions: engineer (1988-1996), postgraduate student (1996-1999), associate professor (2000-2008), Dr Sc (Eng) (2008), professor at the Air Navigation System Department (2009 - to present) at the National Aviation University (Ukraine). Research interests: analysis and synthesis of nonlinear ergatic systems; critical technologies; nonlinear dynamic.

Publications: author of about 70 scientific papers, monographs, textbooks, training aids, articles and proceedings.

Other: managing editor of the Proceedings of the National Aviation University. 


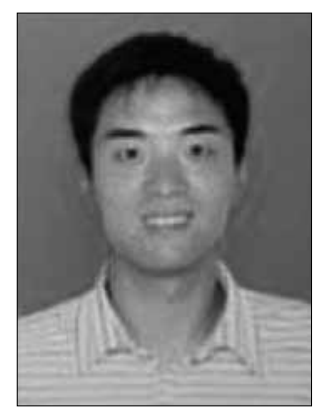

Wang BO, $B S c, M S c, P h D$

Date of birth: 1980.

Education: BSc (1999-2003) in management at Kiev International Civil Aviation

University, MSc (2003-2004) in business operation management at the National Civil

Aviation University, PhD (2005-2009) at the Institute of Logistics of the National Civil

Aviation University.

Affiliations and functions: assistant of the general manager of the marketing department of Easter Airlines (2009-2010), associate professor (2010 - to present) at Port and Logistics

Research Centre co-founded by Ningbo University of Technology and Chinese Academy of Social Sciences.

Research interests: unmanned aerial vehicles; air transportation; cost control; communication and transportation, etc.

Publications: author of 20 papers in core journals and a high-end project and a number of provincial and municipal projects.

\begin{abstract}
A new concept of the synthesis of a synergetic regulator for the control of difficult multidimensional aircraft in polyconflict conditions is offered in the article. The basic idea of control synthesis is the use of self-organization properties of real physical systems for the formation of algorithms for the synergetic regulator operation. This approach allows solving the problem of the high dimensionality of the regulator for aircraft in polyconflict conditions and provides aircraft control in real time. The synergetic approach offered by the authors allows minimising the expenditure of energy for conflict avoidance between aircraft and supports the guaranteed safety level of their motion.
\end{abstract}

Keywords: air traffic management (ATM), polyconflict, synergetic regulator, safety, navigation space.

\section{Introduction}

The safety of modern aircraft depends on the intensity of flights. Specialists forecast an expected growth in air traffic in Europe of 1.6 times until 2025 compared to 2011, and of 2.6 times in 2050 (Flightpath... 2011). Trends in the intensity of flights lead to an increased number of conflicts in which there is an interaction of three or more of the conflicting aircraft, and the notion of "a conflict pair" is transformed into the concept of a multiple conflict (or a polyconflict). The global aviation community is demands the transition from regulated rigid flight paths to free trajectories (Free Flight, 4D), the use of manned aircraft, unmanned and remotely piloted vehicles in a single air navigation space (UAV, RPAS) (Kharchenko, Prusov 2012), the distribution of functions between the pilot and controller with conflict detection and resolution of aircraft (the concept of $\mathrm{A}^{3}$ ), using ADS-B (Action... 2008). At the same time, the requirements concerning safety of both autonomous and controlled traffic services of manned aircraft flight, unmanned and remotely piloted aircraft in a single air navigation space containing static and dynamic exclusion zones and restrictions remain unchanged.

The problem of air traffic safety in modern air navigation space can be solved by creating a single systemic method of polyconflict resolution of an open set of heterogeneous aircraft on short, middle and long spacetime horizons.

Today statistical and probabilistic optimization methods for conflict resolution between aircraft are most commonly used to solve paired conflicts.
Analysis of the application of probabilistic and statistical methods shows that they are not sufficiently effective in the synthesis of conflict-free trajectories of aircraft in cases where:

- The volume of statistical data on the processes occurring in the air navigation environment, when conflicts arise, is small or statistics are completely unavailable.

- Statistical data about conflict characteristics have low reliability.

- Periodic inspections of statistical hypotheses about conflict characteristics are either impossible or involveconsiderable difficulties, etc.

The use of optimization methods has a significant constraint. This is due to the fact that optimization methods are based on the application of a cost function (quality functional). Expanding the cost function by including many parameters leads to a significant increase of size of the controlled process model. The complexity of the controlled process $(N)$ is assessed by complexity of the controlling process (Sage, Mels 1976):

$$
N \geq n+0,5 n(n+1),
$$

where $n$ is the dimensionality of the controlling process.

The analysis of this expression shows that the increase in the complexity of the conflict mathematical model leads to a large increase in the dimension of the conflict-free regulator and creates the effect of the socalled "damnation dimension".

Thus, the use of probabilistic, statistical and optimization methods in many cases does not allow to synthesize aircraft conflict-free trajectories in the event of situations with polyconflicts effectively. 


\section{Review of synergetic approaches}

The complexity of developing an effective systemic method of aircraft polyconflict resolution is associated with the need to solve two major problems - external and internal. The external problem is the need to consider the impact of environmental factors in polyconflict resolution. Participants of these conflicts should collectively consider the external environment in order to self-organize and develop responses aimed at compensation for these impacts. The internal problem is the need to manifest its synergetic properties by the participants of a polyconflict with the purpose to find a safe conflict resolution. The actions of each party should be consistent and in full compliance with the rules to obey.

It seems that it is not possible to solve these problems by standard scientific methods.

The problem solution lies in a synergetic (self-organization) approach to synthesis of air traffic management.

A synergetic approach is not a new one and has been applied in up-to-date research. A well-known approach has been developed by A. Kolesnikov for the synthesis of laws to control nonlinear dynamic objects (Kolesnikov 2000). The approach is based on the procedures of "extension" and "compression" of the phase space. Energy conservation laws are the foundation of the proposed solution. The physical action of the "extension" procedure presumes that the expected movement within the system is provided by sufficient resources (normally these are the energy resources). A sufficient supply of system energy resources provides the system with the capability to functioning appropriately and achieving the goals. The introduction of the energy parameter as an additional phase coordinate into the control model is the backbone of the "extension" procedure of the phase space.

The introduction of additional phase coordinates gives the system new dynamic properties. Under a synthesized synergetic control the extended system reduces the number of available limits of freedom, i.e., it gets asymptotically stable movement to the specified variety of the phase states. "Compression" of the phase space of a nonlinear system is of course the process of system control including its internal nonlinear dynamic properties.

In thermodynamics, the founders of the synergetic approach H. Haken (1991) and I. Prigozhin, D. Kondepuri (2002) distinguish the "order" parameters from a common set of parameters of a complex system. The energy and entropy are the major general-system parameters of the "order", which act as the core of a system's self-organization.

However, due to the difficulty of formalization of the well-known synergetic approaches it is not possible to use them as a general-system basis for the synthesis of air traffic management with polyconflict availability.

\section{Synergetic air traffic management under polyconflict}

Let us conduct the formalization of the synergetic control of aircraft population in polyconflict conditions with the purpose of transforming the disordered conflict system into a system with an expedient dynamics of a conflict-free behavior of each aircraft. Expediency should be understood as the property of transformed processes which provides guaranteed attainability of the specific goal state of each aircraft while ensuring safety for all permissible movements under certain conditions.

The term "guaranteed attainability" by it's meaning is identified with the term "achievable position". If the navigation environment has a property of "guaranteed attainability", then the control is capable off taking out the vector of the phase coordinates of each aircraft from any current position within the navigation environment and putting the system into its position of purpose, in the presence of various perturbations.

To formalize aircraft motion (in a 2-dimensional case) for solving specific applied tasks the following equations are often used:

$$
\begin{aligned}
& \frac{d x_{1 i}}{d t}=f\left(x_{1 i}(t), u_{1 i}(t), \xi_{1 i}(t)\right), \\
& \frac{d x_{2 i}}{d t}=f\left(x_{2 i}(t), u_{2 i}(t), \xi_{2 i}(t)\right), \\
& u_{1 i \min } \leq u_{1 i} \leq u_{1 i \max }, \\
& u_{2 i \min } \leq u_{2 i} \leq u_{2 i \max },
\end{aligned}
$$

where $x_{1 i}, x_{2 i}$ indicate the coordinates of the i-th aircraft; $u_{1 i}, u_{2 i}$ - required conflict-free control of the motion which would ensure their safety and achievement of the goal positions; $\xi_{1 i}, \xi_{2 i}$ - the perturbations acting on the i-th aircraft.

The peculiarity of differential equations (1), taking into account the dynamical processes of aircraft in the presence of multiple conflicts (policonflicts), is that they are essentially a "rolled", "archived" form of the conflict process properties which are inaccessible for direct observation and use. They compose the "external" formal shell of the system under which the "internal" functional properties of the conflict processes are hidden. This leads to the loss of functional and structural properties of a policonflict as well as to a sharp increase of the problem dimension regarding analysis and synthesis of aircraft's motion control under these conditions.

In this paper the authors propose a fundamentally new systemic concept of synergistic control of aircraft in polyconflict condotions, based on the properties of actual force fields. It is understood that:

- the air navigation environment is a big open ergatic (man-machine) system;

- legitimacy, integrity and synergy are the basic properties of the air navigation environment; 
- aircraft have the natural properties of technical systems: the evolution and degradation of the functional state over time;

- integrity and continuity of synergistic dynamic processes of conflicting aircraft control are considered as its systemic invariants.

Most of real world physical systems have unique properties which make them effective. The control processes within the systems are not affected by a "damnation dimension". Besides, the quality of the system's functioning may increase even when expanding the inner subsystems. Moreover, this variety as a rule plays a stabilizing role. Taking into accountthe specified properties of physical systems, it seems to be reasonable and logic to extrapolate the aforementioned properties onaircraft control systems in polyconflict.

The goal of the synthesis of the synergistic regulator polyconflict resolution of population aircraft is to create a virtual world $(\aleph)$ by modeling the properties similar, to some extent, to the properties of a real world physical system. In this case, the properties should ensure the effective operation of the real physical system.

As an example the real limited air navigation space (indicated as $\mathfrak{R}$ ) containing conflicting aircraft, static air space zones forbidden for flights, and also static and dynamic obstacles, such as mountains, storm fronts, etc. will be considered.

In order to form $\aleph$ we use the natural-scientific systemic approach (Chepizhenko et al. 2012), which was most clearly formulated by Helmholtz (Einstein, Infeld 1965): “...the ultimate goal of a physical science is to view the physical phenomena through the inevitable forces of gravitation and repulsion, the power of which completely depends on distance. To approach this problem is equal to understanding the entire nature".

The forces of gravitation and repulsion produced by the force fields serve as the foundation for a synergistic principle of control provision which can be formulated as a "unity of possible and desirable".

The virtual world of $\aleph$ is a complex structure including three subspaces:

- a virtual personal subspace of "desired behaviors" of dynamic objects in $\aleph$;

- a virtual subspace of updated behaviors of dynamic objects in $\aleph$;

- a virtual total subspace in $\aleph$.

To describe the above mentioned subspaces the following principles of the virtual world $\aleph$ are applied:

1 . The world $\aleph$ when considered from the viewpoint of classical mechanics is a complete closed system of static and dynamic objects interacting with each other due to gravitation and repulsion forces acting at any given time point.

2. The world $\aleph$ is formed by virtual strata each corresponding to a dynamic or a static object.
3. Each object immersed in $\aleph$, is a material point with its own mass $m$ and a carrier of a full set of forces (i.e., forces of gravitation and repulsion).

4. The set of forces of any world $\aleph$ object has a property of symmetry. According to classical mechanics, symmetry means that the forces of gravitation and repulsion are acting simultaneously. The forces functionally depend on object mass and on the distance between them.

5. The system of interacting objects in world $\aleph$ is heterogeneous. The objects may be different in their dynamic characteristics and may have different priorities, intentions, sources and goal positions.

6. Static and dynamic constraints can be found in world $\aleph$. They are presented as a "dense" aggregate of material points (with its own mass $m$ ) or as a set of discreet movable material points (with its own mass $m$ ), the motion trajectory of which coincides with the external borders of constraint zones and is formed according to certain algorithms.

7. Terminal goal positions of each dynamic object in world $\aleph$ have a full set of forces (forces of gravitation and repulsion) and are either mobile or fixed material points with their own mass, which is much higher than the mass of dynamic objects.

8. If there are no conflicts, then the desired motion of each dynamic object is performed along the "geodesic" lines of the total force field. The "geodesic" lines are curves in space with arcs for the shortest routes between their ends. The characteristics of the force fields depend on the power potentials and energy put into them.

9. Each dynamic object in $\aleph$ individually identifies its geodesic line with the final point.

The structural and parametric synthesis of the virtual world includes the following steps.

1. The first step in order to formalize the properties of $\aleph$ is to identify its borders as a system of linear inequalities by using the transformation operator $\mathfrak{I}_{\mathfrak{R} \mathcal{K}}^{1}$ :

$$
\Re\left(x_{1}, x_{2}\right)<0 \stackrel{\mathfrak{I}_{\mathfrak{R} \aleph}^{1}}{\Rightarrow} \mathrm{N}\left(z_{1}, z_{2}\right)<0,
$$

where $x_{1}, x_{2}$ indicate the coordinates of $\mathfrak{R}$ borders; $z_{1}, z_{2}$ - the coordinates of $\aleph$ borders.

2. The second step is to transfer multiple positions of closed zones and constraints from $\mathfrak{R}$ into $\aleph$ using the transformation operator:

$$
\begin{gathered}
\mathfrak{R}_{k}\left(x_{1 k}, x_{2 k}\right)<0 \stackrel{\mathfrak{I}_{\mathfrak{R} N}^{2}}{\Rightarrow} \aleph_{k}\left(z_{1 k}, z_{2 k}\right)<0, \\
k \in\left\{1,2, \ldots N_{c}\right\} ; N_{c} \in\left\{N_{r} \cap N_{f} \cap N_{d}\right\} ; \Re_{k} \in \Re ; \\
\aleph_{k} \in \boldsymbol{N},
\end{gathered}
$$

where $x_{1 k}, x_{2 k}$ indicate the coordinates of closed zones and constraints in $\mathfrak{R} ; z_{1 k}, z_{2 k}$ - coordinates of closed zones and constraints in $\aleph ; N_{c}-$ a common set of 
closed zones and constraints in the navigation space; $N_{r}$ - the multitude of rigid closed zones and constraints; $N_{f}$ - the multitude of flexible closed zones and constraints; $N_{d}$ - the multitude of dynamic closed zones and constraints.

3. Thirdly it is required to transfer positions of the aircraft from $\mathfrak{R}$ in $\aleph$ by using the transformation operator $\mathfrak{I}_{\mathfrak{R} X}^{3}$ :

$$
\begin{aligned}
\mathfrak{R}_{i}\left(x_{1 i}, x_{2 i}\right) \stackrel{\mathfrak{I}_{\mathfrak{R} \times}^{3}}{\Rightarrow} \aleph_{i}\left(z_{1 i}, z_{2 i}\right), i \in I\left\{1,2, \ldots N_{\mathrm{AC}}\right\} ; \\
\mathfrak{R}_{i} \notin \mathfrak{R}_{k} ; \aleph_{i} \notin \boldsymbol{\aleph}_{k},
\end{aligned}
$$

where $N_{\mathrm{AC}}$ is the total set of aircraft in a limited navigation space.

4. The fourth step is to transfer goal positions of the aircraft from $\mathfrak{R}$ in $\aleph$ by using the transformation operator $\mathfrak{I}_{\mathfrak{R} X}^{4}$ :

$$
\begin{aligned}
\mathfrak{R}_{i}\left(x_{1 i}^{*}, x_{2 i}^{*}\right) \stackrel{\mathfrak{I}_{\mathfrak{R} \times}^{4}}{\Rightarrow} & \aleph_{i}\left(z_{1 i}^{*}, z_{2 i}^{*}\right), i \in I\left\{1,2, \ldots N_{g}\right\} ; \\
& N_{g} \geq N_{\mathrm{AC}},
\end{aligned}
$$

where $N_{g}$ is the common multitude of aircraft goal positions in a limited navigation space.

5. Afterwards, the procedure is to appoint current positions for each aircraft's $\aleph_{i}\left(z_{1 i}, z_{2 i}\right)$ mass and potentials of repulsion and gravitation:

$$
\begin{gathered}
\forall \aleph_{i}\left(z_{1 i}(t), z_{2 i}(t)\right) \in \boldsymbol{\aleph} \Rightarrow U_{i}^{+}\left(z_{1 i}(t), z_{2 i}(t), r_{i}(t), m_{i}, G\right)+ \\
U_{i}^{-}\left(z_{1 i}(t), z_{2 i}(t), r_{i}(t), r_{\mathrm{cr} i}, m_{i}, G\right) ; \\
i \in I\left\{1,2, \ldots, N_{\hat{\mathrm{D}}}\right\},
\end{gathered}
$$

where $U_{i}^{+}$and $U_{i}^{-}$indicate the potentials of gravitation and repulsion which "accompany" $i$-e aircrafts in $\aleph$ respectively; $r_{i}(t)$ - the distance from the i- $t h$ aircraft; $r_{\text {cr } i}$ - the critical distance from the i-th aircraft; $m_{i}-$ the i-th point mass; $G$ - a constant value of the virtual circuit field, which generates its overall power property in $\aleph ; N_{\mathrm{DO}}$ - the number of dynamic objects.

The critical distance between the points in $\aleph$ is specified on account of regulatory requirements in their safety zones. The condition needed for the appointment of aircraft safety zone size in $\mathfrak{R}$ is as follows:

$$
r_{c r i} \geq \max \left(\Delta x_{1 \mathrm{i}}, \Delta x_{2 \mathrm{i}}\right) \text {, }
$$

where $\Delta x_{1 \mathrm{i}}, \Delta x_{2 \mathrm{i}}$ indicate the geometric dimensions of the $i$-th aircraft.

6. The potentials of repulsion and gravitation are set to the goal (terminal) positions $\aleph_{i}\left(z_{1 i}^{*}, z_{2 i}^{*}\right)$ of the $i$-th aircraft:

$$
\begin{gathered}
\forall \aleph_{i}\left(z_{1 i}^{*}(t), z_{2 i}^{*}(t)\right) \in \aleph \Rightarrow U_{i}^{+}\left(z_{1 i}^{*}(t), z_{2 i}^{*}(t), r_{i}^{*}(t), m_{i}^{*}, G\right)+ \\
U_{i}^{-}\left(z_{1 i}^{*}(t), z_{2 i}^{*}(t), r_{i}^{*}, m_{i}^{*}, G\right) ; \\
\quad i \in I\left\{1,2, \ldots N_{\mathrm{g}}\right\},
\end{gathered}
$$

where $z_{1 i}^{*}, z_{2 i}^{\star}$ indicate the coordinates of the $i$-th aircraft terminal position; $r_{i}^{*}(t)$ - the distance from the $i$-th aircraft goal position, $m_{i}^{*}$ is the mass of aircraft goal (terminal) positions.
7. Closed zones and constraints of the real navigation space are displayed in $\aleph$ as geometrical primitives (circle, ellipse, line, etc.) or their match. The boundaries of closed zones are presented as a finite multitude of points each of which is defined by the potentials of gravitation and repulsion:

$$
\begin{gathered}
\forall \aleph_{k}\left(z_{1 k}(t), z_{2 k}(t)\right) \in \boldsymbol{\aleph} \Rightarrow U_{k}^{+}\left(z_{1 k}(t), z_{2 k}(t), r_{k}(t), m_{k}, G\right)+ \\
U_{k}^{-}\left(z_{1 k}(t), z_{2 k}(t), r_{k}(t), r_{\text {cr } k}, m_{k}, G\right) ; \\
k \in\left\{1,2, \ldots N_{\tilde{n}}\right\},
\end{gathered}
$$

where $N_{\tilde{n}}$ indicates the number of points forming all closed zones in $\aleph$.

8. Each point of the $\aleph$ space is characterized by the total potential of the artificial force field:

$$
\begin{aligned}
& U_{\Sigma}\left(z_{1}, z_{2}\right)=\sum_{i=1}^{N_{\mathrm{AC}}}\left(U_{i}^{+}\left(z_{1 i}(t), z_{2 i}(t), r_{i}(t), m_{i}, G\right)+\right. \\
& \left.U_{i}^{-}\left(z_{1 i}(t), z_{2 i}(t), r_{i}(t), r_{\mathrm{cr} i}, m_{i}, G\right)\right)+ \\
& \sum_{i=1}^{N_{\mathrm{g}}}\left(U_{i}^{+}\left(z_{1 i}^{*}(t), z_{2 i}^{*}(t), r_{i}^{*}(t), m_{i}^{*}, G\right)+\right. \\
& \left.U_{i}^{-}\left(z_{1 i}^{*}(t), z_{2 i}^{*}(t), r_{i}^{*}, m_{i}^{*}, G\right)\right)+ \\
& N_{\mathrm{c}}\left(U_{k}^{+}\left(z_{1 k}(t), z_{2 k}(t), r_{k}(t), m_{k}, G\right)+\right. \\
& \sum_{k=1} \\
& \left.U_{k}^{-}\left(z_{1 k}(t), z_{2 k}(t), r_{k}(t), r_{\mathrm{cr} k}, m_{k}, G\right)\right) .
\end{aligned}
$$

Each object put in $\aleph$, generates the resentment of the total artificial force field and is characterized by its virtual potentials of gravitation and repulsion:

$$
U_{i}^{+}=\frac{G m_{i}}{r_{i j}}, U_{i}^{-}=-\frac{G m_{i}}{r_{i j}} .
$$

The resultant force vector at each $\aleph$ point has the forces of gravitation and repulsion:

$$
\begin{aligned}
& F_{i j}^{+}=\frac{G m_{i} m_{j}}{r_{i j}^{\alpha}}=U_{i}^{+} \frac{m_{j}}{r_{i j}^{(\alpha-1)}} ; \\
& F_{i j}^{-}=\frac{G m_{i} m_{j} r_{\mathrm{cr}}}{r_{i j}^{\beta}}=U_{i}^{-} \frac{m_{j} r_{\mathrm{cr}}}{r_{i j}^{(\beta-1)}} ; 1<\alpha<\beta,
\end{aligned}
$$

where $\alpha, \beta$ indicates natural numbers.

The corresponding projections of the gravitation and repulsion forces on axis $z_{1}$ and $z_{2}$ are as follows:

$$
\begin{aligned}
& F_{i j z 1}^{+}=F_{i j}^{+} \frac{\left|z_{1 i}-z_{1 j}\right|}{r_{i j}} ; F_{i j z 2}^{+}=F_{i j}^{+} \frac{\left|z_{2 i}-z_{2 j}\right|}{r_{i j}} ; \\
& F_{i j z 1}^{-}=F_{i j}^{-} \frac{\left|z_{1 i}-z_{1 j}\right|}{r_{i j}} ; F_{i j z 2}^{-}=F_{i j}^{-} \frac{\left|z_{i}-z_{j}\right|}{r_{i j}} ; \\
& r_{i j}=\sqrt{\left(z_{1 i}-z_{1 j}\right)^{2}+\left(z_{2 i}-z_{2 j}\right)^{2}} .
\end{aligned}
$$


In equations (2) and (3), the aggregate virtual world environment (synergistic substance) is selected according to the ratio of $\alpha / \beta$. This state of the environment characterizes the degree of self-organization of aircraft in a policonflict. The analogical state of the aggregate virtual environment is aggregate physical substances, e.g., gaseous, liquid, crystal, etc.

According to Newton's second law, a motion of material points, endowed by masses formed in the above artificial force field, is described by the following equations:

$$
\frac{d^{2} z_{1 i}}{d t^{2}}=\frac{1}{m_{i}}\left(\sum_{j=1}^{N_{\mathrm{AC}}+N_{\mathrm{ob}}+N_{\mathrm{g}}} F_{i j}^{z_{1}+}+\sum_{j=1}^{N_{\mathrm{AC}}+N_{\mathrm{ob}}+N_{\mathrm{g}}} F_{i j}^{z_{1}-}+\mu_{1} \frac{d z_{1 i}}{d t}\right)
$$$$
\frac{d^{2} z_{2 i}}{d t^{2}}=\frac{1}{m_{i}}\left(\sum_{j=2}^{N_{\mathrm{AC}}+N_{\mathrm{ob}}+N_{\mathrm{g}}} F_{i j}^{z_{2}+}+\sum_{j=2}^{N_{\mathrm{AC}}+N_{\mathrm{ob}}+N_{\mathrm{g}}} F_{i j}^{z_{2}-}+\mu_{2} \frac{d z_{2 i}}{d t}\right)
$$

$$
\forall j \neq i
$$

where $N_{\mathrm{ob}}$ indicates the number of static and dynamic obstacles; $\mu_{1} \frac{d z_{1 i}}{d t}$ and $\mu_{2} \frac{d z_{2 i}}{d t}$ are damping compon-
ents.

As a result of the integration of equations in (4), the direction of the velocity vector of each material point, which determines synergistic conflict-free aircraft control under a polyconflict, can be identified:

$$
u_{i s}=\psi_{i}(k)=\operatorname{arctg}\left(\frac{z_{2 m_{i}}(k)-z_{2 m_{i}}(k-1)}{z_{1 m_{i}}(k)-z_{1 m_{i}}(k-1)}\right),
$$

where $k$ is the integration step of the system of equations in (4).

When forming synergistic conflict-free aircraft control the following limitations must be considered:

$$
\frac{d \psi_{i}}{d t}=\frac{g}{V_{i}} \operatorname{tg} \gamma_{i \lim }
$$

where $g$ is constant; $\gamma_{i \text { lim }}$ indicates a limitation of the roll angle current value of $i$-th aircraft depending on the flight mode and conditions.

The common law of aircraft control by using a synergistic regulator has the following form:

$$
u=u_{g}+u_{s}
$$

where $u_{g}$ indicates the goal component of aircraft vector control, $u_{s}$ - the synergistic component of vector conflict-free aircraft control.

A block diagram of the aircraft control system using a synergistic regulator based on the aforementioned principles is illustrated in figure 1.

The principal feature and advantage of the formed virtual world $\aleph$ is that when the material points are approaching the critical distance $r_{\mathrm{cr}}$, the resulting force acting on them is equal to zero. In other words, the attractive and repulsive forces balance each other (Chepizhenko et al. 2013). At a distance $r_{\mathrm{cr}}$ the energy of ma-

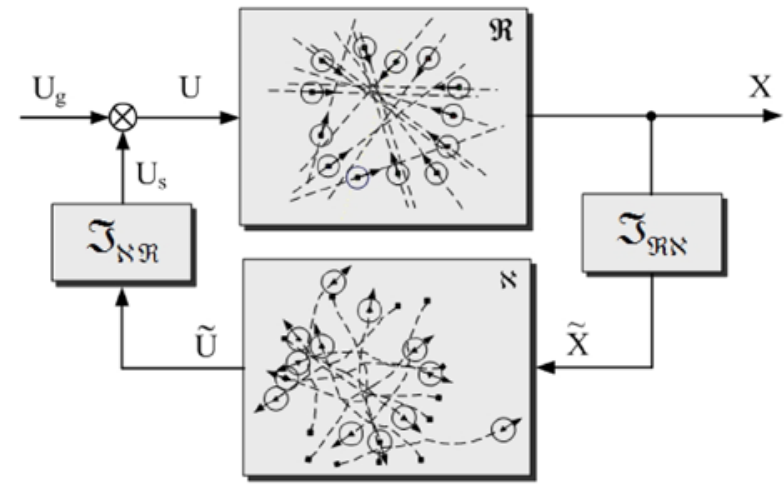

Fig. 1. Block diagram of synergistic aircraft control

terial point interaction reaches its global minimum, i.e., the least interaction between the physical bodies. Alongside, distance $r_{\text {cr }}$ allows specifying the dimensions of safety zones for the aircraft. The absence of intersections of such zones under the condition of unpredictability of aircraft position makes it possible to maintain guaranteed level of safety of the element motion in situations of policonflict.

The above mentioned principles form $\aleph$ as a common field model of navigation space, free of heuristics and incompleteness of functional supply. This model does not depend on the quantity of objects in $\aleph$ (taking into account limits of its full capacity), and allows:

- forming the properties of the artificial force field in $\aleph$ as a general topological model of navigation space;

- simultaneously synthesizing a conflict-free synergistic control for all aircraft involved in the conflict;

- minimizing the complexity of the navigation space model and excluding the "damnation dimension".

During the synthesis of conflict-free trajectories only the command track angle is calculated, and the value of the velocity vector of the aircraft is not changed. Therefore, only the length of the synthesized trajectory from the starting point of a polyconflict resolution until the aircraft's goal point (criterion (6)) acts the fuel consumption rate in the horizontal plane.

The efficiency rating of the use of synergistic aircraft control is carried out according to the criterion of minimum relative aircraft track $S_{\text {rel }}$ from the starting position to the finishing position in terms of obstacle avoidance with a set geometry:

$$
S_{\text {rel }}=\frac{S_{\text {sim }}}{S_{\text {sh }}}
$$

where $S_{\text {sim }}$ indicates a real (calculated in the simulation) aircraft track from the start point to the end (terminal) point; $S_{\text {sh }}$ - the shortest distance from the starting point of the aircraft to the goal point. 
Only the graphic data on the length of the trajectories synthesized by the methods of solution of dual conflicts in the available literature (Chaloulos et al. 2009; Eby 1994; Hwang, Tomlin 2002; Zakora 2006) is available. Therefore, comparison of the efficiency of synergistic aircraft control carried out with these methods of dual conflict resolution of aircraft collisions according to criterion (6) is carried out.

The simulation results indicate that the use of synergistic aircraft control to resolve conflicting aircraft collisions can reduce the value of criterion (6) up to $5 \%$ compared to other existing methods (Fig. 2) of the resolution of dual conflicts.

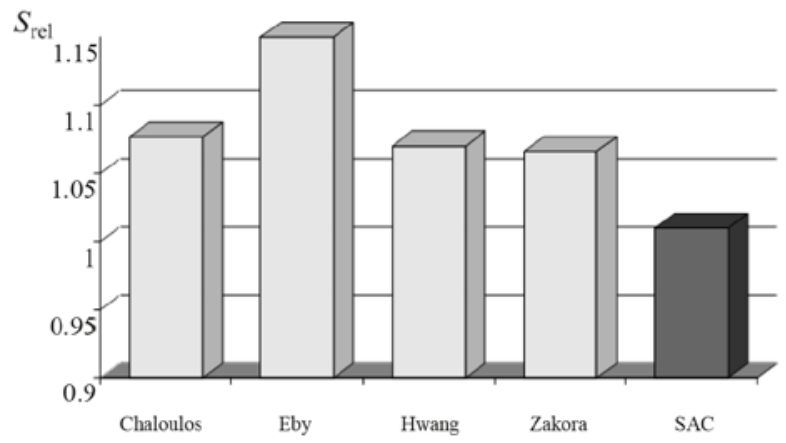

Fig. 2. The value of criterion (3.1) for methods of dual conflict resolution, considered in the references (Chaloulos et al. 2009; Eby 1994; Hwang, Tomlin 2002; Zakora 2006) and for synergistic aircraft control (SAC)

The concept of synergistic aircraft control also allows synthesizing an effective conflict-free trajectory by other criteria, such as simplicity trajectory pilot, comfort trajectory based on the needs of passengers or cargo, etc. However, the data about the values of these criteria for other methods of conflict resolution aircraft are missing. Therefore, the comparison was not carried out.

\section{Conclusions}

The proposed concept addresses the solution of the problem of increasing the current level of flight safety in the areas under responsibility of ground control centers in the medium CNS/ATM through operational and strategic conflict prediction and synthesis of real-time effective conflict-free trajectories of aircraft motions in the free flight zones ("Free Flight"). The concept may be the basis for developing intelligent automated ground air traffic management systems and aircraft on-board control in difficult conditions, at high air traffic intensity and in situations of polyconflict.

It is expected that the proposed concept will allow to:

- Solve the problem of the "damnation dimension" in complex conflict resolution of collision of aircraft open set.
- Carry out a simultaneous polyconflict resolution without structuring to "paired components" that avoids the "domino effect".

- Synthesize in real-time effective conflict-free trajectories of aircraft motions in 4D space.

- Minimize expenditure of energy for implementing conflict-free trajectories of aircraft motions.

- Ensure the autonomy of the control loops of flight speed and angular positions of the velocity vector in the synthesis of conflict-free trajectories of aircraft.

- Consider the priority of aircraft based on their functional tasks and their functional state.

- Synthesize conflict-free purposeful motion paths for different classes of dynamic objects such as manned and unmanned aerial vehicles, ground moving vehicles, mobile robots, autonomous surface and underwater vehicles.

The expected impact is as follows:

- Enhanced reliability of the decision-making process in complex distributed systems of air traffic management.

- Increased flight safety.

- Improved economic efficiency of air traffic services, including the modes (zones) of free flights.

- Optimized air navigation space.

- Noticeably increased safety of aircraft density in air navigation space.

\section{References}

Action Plan 23: Long term ADS-B and ASAS Applications. 2008. D3 - Operational Role of Airbone Surveillance in Separating Traffic. FAA/EUROCONTROL cooperative R\&D. $105 \mathrm{p}$.

Chaloulos, G.; Roussos, G. P.; Lygeros, J., et al. 2009. Mid and short term conflict resolution in autonomous aircraft operation, in 8th Innovative Research Workshop \& Exhibition, December 2009, Bretigny-sur-Orge, France, 1-6.

Chepizhenko, V.; Kharchenko, V.; Pavlova, S. 2013. Synergy of piloted, remotely piloted and unmanned air systems in single air navigation space, Logistics and Transport 2(18): 77-82.

Chepizhenko, V. I.; Pavlov, V. V.; Pavlova, S. V. 2012. Virtual Einsteinian force fields in a synergy of navigating space of difficult ergatic systems, Proceedings of National Aviation University 3: 15-27.

Eby, M. S. 1994. A self-organizational approach for resolving air traffic conflicts, The Lincoln Laboratory Journal 7(2): 239-254.

Einstein, A.; Infeld, L. 1965. Physics Evolution. Moscow: Science. 296 p. (in Russian).

Flightpath 2050. 2011. Europe's vision for aviation, in Report of the High-Level Group on Aviation Research. Luxembourg: Publications Office of the European Union. 32 p.

Haken, H. 1991. The Information and Self-Organization: the Macroscopic Approach to Difficult Systems. Moscow: Mir. 240 p. (in Russian). 
Hwang, I.; Tomlin, C. J. 2002. Protocol-Based Conflict Resolution for Air Traffic Control. USA, Stanford: Department of Aeronautics and Astronautics Stanford University. 49 p.

Kharchenko, V.; Prusov, D. 2012. Analysis of unmanned aircraft systems application in the civil field, Transport 27(3): 335-343. http://dx.doi.org/10.3846/16484142.2012.721395

Kolesnikov, A. A. (Ed). 2000. Modern Applied Theory of Control: the Synergetic Approach in the Control Theory. Part. II. Taganrog: Publishing house TRTU. 559 p. (in Russian).

Prigozhin, I.; Kondepuri, D. 2002. Modern Thermodynamics: From Thermal Engines to Dissipative Structures. Moscow: Mir. 464 p. (in Russian).

Sage, A.; Mels, Dj. 1976. The Estimation Theory and its Application in Communication and Control. Moscow: Svyaz. 495 p. (in Russian).

Zakora, S. A. 2006. Group Conflict Situations Resolution on Free Routes in Terms of the Guaranteed Level of Safety: Dis. of candidate of technical sciences. 141 p. (in Ukrainian). 